\title{
Combining Hydraulic and Electric Actuations To Improve Efficiency and Control Effectiveness for Off-Road Mobile Machines
}

\author{
Perry Y. Li \\ Department of Mechanical Engineering \\ University of Minnesota \\ Minneapolis, MN 55455 \\ USA
}

Off-road mobile machines, such as excavators and wheel-loaders are typically actuated using hydraulics because of the high power requirements. Because of the reliance on throttling valves for control and the inability to recuperate energy from overrunning loads, the efficiencies of such machines from engine output to useful work is only about $21 \%$. Yet off-road vehicles are responsible for as much as $1.0 \%$ of all energy consumed in the U.S.A.. Therefore, similar to on-road vehicles, two current trends for off-road mobile machines are towards improved efficiency and electrification. An issue with electrifying off-road vehicles is the high power requirements and the high cost for high power electrical components. In this talk, I will discuss a new throttle-less and regenerative system architecture, known as Hybrid Hydraulic-Electric Architecture (HHEA), which combines the powerdensity advantage of hydraulic and the efficiency and controllability advantages of electric actuation. In particular, the majority power is transmitted hydraulically while electric actuation is used only to modulate this power. This way, the power requirement for electrical components can be kept small. I will highlight interesting control problems for this architecture including optimal power flow (OPF) to maximize energy savings, and control design for precise motion control in the presence of discrete pressure switches. Preliminary investigation indicates that the new architecture can reduce energy consumption by two-thirds compared to the baseline load-sensing architecture. 